\title{
Functional Electrical Stimulation (FES) Reduces Intermittent Claudication (IC) and Improves Quality of Life (QOL) Measures: A Cohort Crossover
}

\author{
Alon $\mathrm{G}^{1 *}$, Embrey DG ${ }^{2}$, Brandsma BA $^{2}$, Vladimir \\ $F^{3}$, Silva $A^{2}$, Pflugeisen $B^{2}$, Sherif $D^{2}$ and \\ Amoroso PJ $^{2}$ \\ ${ }^{1}$ Department of Physical Therapy \& Rehabilitation \\ Science, University of Maryland, School of Medicine, \\ Baltimore, USA \\ ${ }^{2}$ MultiCare Institute for Research and Innovation, \\ Tacoma WA, USA \\ ${ }^{3}$ Swedish Hospital, Seattle WA, USA \\ *Corresponding author: Gad Alon, Department of \\ Physical Therapy \& Rehabilitation Science, University \\ of Maryland, School of Medicine, 100 Penn Street, \\ Baltimore, MD 21201, USA
}

Received: September 29, 2017; Accepted: October 18, 2017; Published: October 25, 2017

\begin{abstract}
Aim: This crossover investigation applied functional electrical stimulation (FES) to the dorsiflexors (DF) and plantarflexors (PF) during walking in the home environment, hypothesizing that it will reduce intermittent claudication (IC), improve walking distance, and enhance quality of life measures compared to three baseline data points during which FES was not provided.
\end{abstract}

Methods: Nine male patients, $68.4 \pm 4$ yrs old, and ankle-brachial index between 0.4-0.8 who completed 16 weeks walking $1 \mathrm{hr}, 6$ days/week without FES consented to walk the same amount wearing FES applied bilaterally to the DF and PF. Measurements of perceived pain intensity (PPI) at the end of a 6 min walk test $(6 \mathrm{MW})$, walking distance, peripheral arterial disease quality of life (PADQOL), intermittent claudication questionnaire (ICQ) and timed up and go (TUG) were obtained at baseline (T0), after 8 weeks walking without FES (T1), after 8 weeks follow up (T2) and after 8 weeks walking with FES (T3). Repeated ANOVA was used to analyze the data and significance was assessed at the $\mathrm{P}<.05$ level.

Results: PPI at T0 was $51.3 \pm 19.4$ and significantly dropped to $9.9 \pm 11.1$ $(p<.001)$ at T3 registering a large effect size $(\eta 2=.42)$, PADQOL factor 3 improved by 14.8 points between T0 and T3 $(\mathrm{p}=.01 ; \eta 2=.36)$ and ICQ score improved by 16.3 points $(p=.004 ; \eta 2=.36)$. The $6 \mathrm{MW}$ distance and TUG did not change during the study.

Conclusion: This crossover study added validity to the hypothesis that applying FES while walking can markedly diminish the intermittent claudication pain and improve quality of life measures in community ambulatory patients with PAD and IC.

Keywords: Intermittent Claudication; FES; Pain; Walking

\section{Introduction}

Functional electrical stimulation (FES) is a common intervention option in rehabilitation medicine supported by robust peer-reviewed publications [1-4]. With the advent of biomedical electronics, the adoption of wireless wearable FES systems is gaining acceptance in clinical practice over the more traditional, non-wearable, wiredependent devices collectively known as neuromuscular electrical stimulators (NMES) [4]. The primary advantage of wearable FES systems is enabling many patients to improve performance in activities of daily living (ADL). Several investigators tested the benefit of using wearable FES on improving the ability to walk following damage to the brain. Springer and colleagues [5] reported that after training for 6 weeks using a dual channel wireless FES, subjects with limited household ambulation capabilities improved their gait speed by $63.3 \%$, while subjects with functional community ambulation capabilities improved their gait speed by $25.5 \%$. Kluding et al examined the benefits of walking for 30 weeks with a wireless FES designed to control foot drop associated with damage to the brain, comparing it to articulated ankle-foot orthosis (AFO), and concluded that the FES yielded clinically and statistically significant improvements in gait speed and other functional outcomes including greater patient satisfaction with using the FES compared to using the AFO [6]. These advanced FES systems to control foot drop are also benefiting pediatric patients with brain damage after daily use for at least a year [7].

Before wearable wireless FES systems were commercially available, wires-dependent non-invasive FES were applied during daily activities to help patients suffering from stroke, multiple sclerosis (MS), traumatic brain injury (TBI), or Parkinson's disease, and a number of investigators reported improved locomotion while using wire-dependent FES [1,8-11] and less favorable outcomes using only AFO [12]. Moreover, numerous clinical studies using NMES to induce muscle contraction in the absence of functional training, typically limit clinical outcome to minimize impairments. Specifically, clinical data support the use of NMES to significantly increase total RNA content and reduce protein degradation in post-operative patients [13], improve physical performance, partially reverse sarcopenia by increasing muscle cross section area and capacity to perform daily activity $[4,14]$, and increase the body's glycolytic metabolism in patients with type-2 diabetes mellitus $[15,16]$.
Phys Med Rehabil Int - Volume 4 Issue 5 - 2017 ISSN : 2471-0377 | www.austinpublishing group.com Alon et al. () All rights are reserved 
Applying wearable FES to patients with peripheral arterial disease (PAD) while walking is a novel approach aimed at reducing intermittent claudication (IC), improving walking distance, and enhancing quality of life measures. We recently published a manuscript [17] detailing the effects of walking for one hour, six days each week, for eight weeks on three primary outcome measures: the perceived pain intensity (PPI) at the completion of the 6-min (6MW) walk test, the distance walked in six minutes, and the Peripheral Arterial Disease Quality of Life (PADQOL) measures. One group using FES while walking (FES+Walk) was compared to a group walking at the same prescribed dose, but without FES (Walk). Both groups were followed for additional 8 weeks after completing the 8 -week training. The FES+Walk group experience marked drop in IC (PPI score) dropping 65.5\% at the end of the follow-up, while the WALK group PPI scores increased $8 \%$ in the same time period. The walking distance of the FES+Walk increased by 42.2 meters (14.5\%) compared to only 24.4 meters (8.5\%) and the factor 3 of PADQOL score of the FES+Walk group increased by 12.9 points compared to only 5.1 points of the Walk group. At the completion of the follow up period, patients in Walk only group were offered the FES to apply at home for 8 weeks thus enabling to extend the study as a crossover design. The aim was to test if the benefits of FES+walk reported in the original study could be reproduced. The specific hypothesis of this crossover investigation was that applying FES to the dorsiflexors (DF) and plantarflexors (PF) during walking in the home environment will reduce intermittent claudication, improve walking distance, and enhance quality of life compare to the three baseline data points in which FES was not provided.

\section{Methods}

Patients in this study $(\mathrm{N}=9)$ chose to participate in the crossover phase and receive FES. The crossover protocol was approved by the local institutional review board. Of the five patients choosing not to continue participation, three stated they are walking fine and not interested in FES, one developed foot ulcer and was unable to walk and one patient declined due to financial hardship. Participating patients were ambulatory, community dwelling adults diagnosed with PAD and IC with an Ankle Brachial Index (ABI) between 0.4 and 0.8. All patients received standard care by their primary physicians, defined as conservative (non-surgical) management of signs and symptoms of $\mathrm{PAD}$, and prescribing appropriate medications to including those to improve circulation and manage pain.

\section{Outcome measures}

Baseline (T0), post-treatment (T1) and follow up (T2) measures were collected according to the protocol from the original study [17]. The crossover data (T3) documented outcome measures for the nine patients enrolled in the crossover phase. Five measures included: 1) Walking distance was determined by measuring meters traveled during the $6 \mathrm{MW}$ test [18]. If patients were unable to walk the full six minutes, they were allowed to sit and rest until they were ready to continue. 2) The PPI was used to determine the severity of ischemic walking pain. After the $6 \mathrm{MW}$, investigators asked patients to rate their ischemic pain in their lower legs by placing a hatch mark on a $0-100 \mathrm{~mm}$ horizontal line. Patients were instructed that 0 meant "no pain" and 100 meant "the most intense pain ever experienced." 3) Changes in lifestyle were measured using Factor 3 (Symptoms and Limitations in Physical Functioning) of the Peripheral Arterial Disease

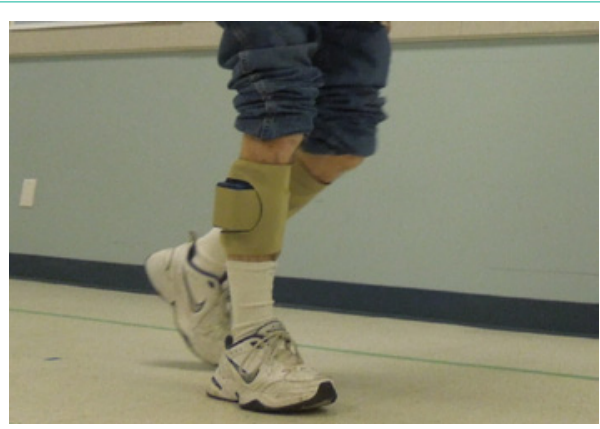

Figure 1a: A prototype FES system designed to activate the dorsiflexors and plantarflexors bilaterally while walking.

Quality of Life (PADQOL) questionnaire [19]. 4) The Intermittent Claudication Questionnaire (ICQ) measured the impact of walking pain on quality of life. This measure has been shown to be a reliable and valid measure of quality of life for patients with IC [20]. 5) The Timed Up and Go (TUG) measured the time (sec) needed for patients to stand up from a standard chair, walk 3 meters, walk around a cone, return to the chair, and sit down. A video camera perpendicular to the walkway recorded this task to assure accurate data collection.

\section{Intervention procedures}

Patients were instructed to walk one hour per day, six days per week, in their natural environment while using the FES system during the 8-week phase. Patients kept a daily log to document walking time per day, physician visits, medication changes, and any unforeseen complications. Weekly visits provided the investigator an opportunity to review daily logs, respond to questions, and monitor FES application.

\section{FES system}

The FES system provided electrical stimulation to the DF and PF while the patient was walking (Figure 1a). Each FES unit contained a motion sensor that automatically triggered muscle contractions of the PF ( $40 \%$ of the gait cycle) and DF (60\% of the gait cycle). Customized software allowed adjustments in the intensity ( $\mu$ coul) and pulses/s (PPS) of each FES unit. This specific FES system delivered symmetrical biphasic waveform pulses, at a rate of 25PPS to the DF and 8-15 PPS to the PF. Stimulation intensity for each patient was adjusted by increasing the phase charge ( $\mu$ coul) of each channel.

On each leg, two surface electrodes of $5 \times 5 \mathrm{~cm}$ were placed over the DF while one $8 \times 12 \mathrm{~cm}$ electrode and one $5 \times 9 \mathrm{~cm}$ electrode were placed over the PF. Electrodes were attached with Velcro to the inside of a neoprene cuff which contained the FES unit. Patients used a remote control for each leg to turn the remote on/off, select WALK mode and adjust the intensity (Figure $1 b$ ).

\section{Data analysis}

Descriptive statistics and data visualizations for each measure at each time point were examined. Repeated measures ANOVA were used to assess the factor of time for each metric during the control period alone (T0 - T2) and including the crossover period (T3) for the subset of nine patients who opted to participate in the crossover phase. All assumptions were closely examined, including sphericity and homogeneity of variance/covariance matrices. In the event that the assumption of sphericity was violated, $\mathrm{p}$-values were 
Table 1: Descriptive statistics $(n=9)$ and repeated measures ANOVA results for each measure at each time point.

\begin{tabular}{|c|c|c|c|c|c|c|c|}
\hline \multirow[b]{2}{*}{ Measure } & \multirow[b]{2}{*}{ Time $^{\dagger}$} & \multicolumn{4}{|c|}{ Descriptive Statistics } & \multicolumn{2}{|c|}{ Repeated Measures ANOVA } \\
\hline & & Mean & Range & $\mathrm{SD}$ & $95 \% \mathrm{Cl}$ & $\begin{array}{c}\text { Control Phase } \\
\left(T_{0}-T_{2}\right)\end{array}$ & $\begin{array}{c}\text { Including Crossover } \\
\left(\mathrm{T}_{0}-\mathrm{T}_{3}\right)\end{array}$ \\
\hline \multicolumn{8}{|c|}{ Primary Measures } \\
\hline \multirow{4}{*}{ PPI } & T0 & 51.3 & $25-75$ & 19.4 & $38.7-64.0$ & \multirow{4}{*}{$\mathrm{p}=.59$} & \multirow{4}{*}{$\begin{array}{l}\mathrm{p}^{*}<.001 \\
\mathrm{\eta}^{2}=.42\end{array}$} \\
\hline & $\mathrm{T} 1$ & 47.8 & $4-83$ & 28.1 & $29.4-66.1$ & & \\
\hline & $\mathrm{T} 2$ & 54.2 & $20-89$ & 26.6 & $36.8-71.6$ & & \\
\hline & T3 & 9.9 & $0-28$ & 11.1 & $2.7-17.1$ & & \\
\hline \multirow{4}{*}{$6 \mathrm{MW}$} & T0 & 317.7 & $194-387$ & 65.2 & $275-360.3$ & \multirow{4}{*}{$\mathrm{p}^{*}=.37$} & \multirow{4}{*}{$\begin{array}{l}p^{*}=.14 \\
\eta^{2}=.06\end{array}$} \\
\hline & $\mathrm{T} 1$ & 329.3 & $213-378$ & 52.7 & $294.9-363.8$ & & \\
\hline & $\mathrm{T} 2$ & 335.1 & $237-376$ & 45.0 & $305.7-364.5$ & & \\
\hline & T3 & 353.7 & $237-415$ & 51.9 & $319.7-387.6$ & & \\
\hline \multirow{3}{*}{ PADQOL } & $\mathrm{T} 1$ & 16.8 & $9-30$ & 6.3 & $12.7-20.9$ & \multirow{3}{*}{$p=.10$} & \multirow{3}{*}{$\begin{array}{l}\mathrm{p}^{*}=.01 \\
\eta^{2}=.36\end{array}$} \\
\hline & $\mathrm{T} 2$ & 18.2 & $10-35$ & 7.4 & $13.4-23.1$ & & \\
\hline & T3 & 29.7 & $15-48$ & 11.4 & $22.2-37.1$ & & \\
\hline \multicolumn{8}{|c|}{ Secondary Measures } \\
\hline \multirow{4}{*}{$\mathrm{ICQ}$} & T0 & 49.8 & $37-60$ & 8.1 & $44.5-55.0$ & \multirow{4}{*}{$\mathrm{p}=.28$} & \multirow{4}{*}{$\begin{array}{l}\mathrm{p}^{*}=.004 \\
\eta^{2}=.36\end{array}$} \\
\hline & $\mathrm{T} 1$ & 52.0 & $37-68$ & 9.9 & $45.5-58.5$ & & \\
\hline & $\mathrm{T} 2$ & 52.4 & $37-65$ & 9.8 & $46.1-58.8$ & & \\
\hline & T3 & 66.1 & $53-79$ & 8.2 & $60.7-71.5$ & & \\
\hline \multirow{3}{*}{ TUG } & T0 & 10.2 & $8.9-11.2$ & 0.8 & $9.7-10.7$ & \multirow{3}{*}{$\mathrm{p}=.86$} & \multirow{3}{*}{$\begin{array}{c}p=.88 \\
\eta^{2}=.01\end{array}$} \\
\hline & $\mathrm{T} 1$ & 10.2 & $7.9-13.1$ & 1.5 & $9.2-11.2$ & & \\
\hline & $\mathrm{T} 2$ & 10.0 & $8.6-11.4$ & 1.0 & $9.3-10.6$ & & \\
\hline
\end{tabular}

PPI: Perceived Pain Index; 6MW: Six Minute Walk Test; TUG: Timed Up-and-Go; PAD-QOL: Factor three of the PAD-QOL questionnaire; ICQ: Intermittent Claudication Questionnaire.

${ }^{\dagger} \mathrm{T}_{0}-\mathrm{T}_{2}$ comprises the control phase of the study in which patients did not use the GMES; $\mathrm{T}_{3}$ : indicates the crossover phase.

$\mathrm{p}^{*}$ : indicates the $\mathrm{p}$-value was adjusted using the Greenhouse-Geisser correction for the violation of the assumption of sphericity.

$\eta^{2}$ : Generalized eta-squared measure of effect size.

adjusted using the Greenhouse-Geisser correction. Significance was assessed at the $\mathrm{P}<.05$ level; Greenhouse-Geisser corrected $\mathrm{p}$-values are denoted throughout the manuscript with $\mathrm{p}^{*}$. All analyses were conducted using SPSS v22.0 (Armonk, New York) and the R Statistical Computing (Vienna, Austria).

\section{Results}

The nine patients in the crossover cohort were all male, had an average age of $68.4 \pm 4.0$ years and a mean BMI of $28.0 \pm 4.7 \mathrm{~kg} / \mathrm{m}^{2}$. At baseline, mean lowest $\mathrm{ABI}$ was $0.60 \pm 0.12$ and the patients were evenly distributed across PAD severity categories, with three patients having moderate, three patients severe, and three patients profound Perceived Pain Intensity (PPI). At the end of 8 weeks walking with the FES, all were in the mild pain category. The average number of walking minutes without FES each day (logged by each patient) of the original study was $51.9 \pm 19.1 \mathrm{~min}$ and while walking with FES for 8 weeks it increased to $65.8 \pm 47.5 \mathrm{~min}$. Of the nine subjects, three increased walking time from $26 \%$ to $106 \%$ while five improved slightly or did not change, and one decreased walking time by $39 \%$.

Descriptive and analytical data for each outcome measure at each time point are summarized in Table $1 . \mathrm{T} 0, \mathrm{~T} 1$, and $\mathrm{T} 2$ represent the testing time points when participants were in the control (walk only) group of the original study and T3 represent the testing time point following 8 weeks of walking with the FES. At study completion, the group's mean IC measured by the PPI was reduced by $81.7 \%$, the distance walked in $6 \mathrm{~min}$ increased by $5.5 \%$, factor 3 of the PADQOL increased by $63.2 \%$ and the ICQ by $26.1 \%$. Performance on the TUG remained unchanged after 8 weeks of walking with the FES.

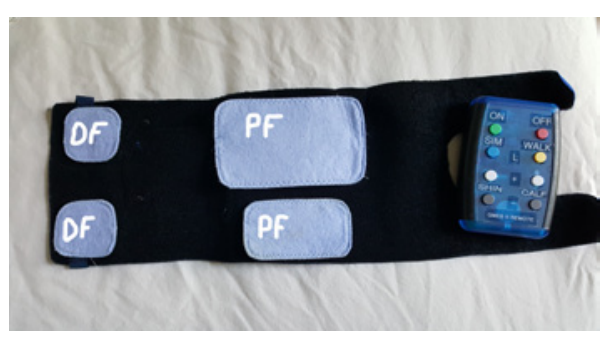

Figure 1b: Water based microfiber electrodes cuff and a wireless remote control.

Footnote: DF: Dorsiflexors; PF: Plantarflexors. 

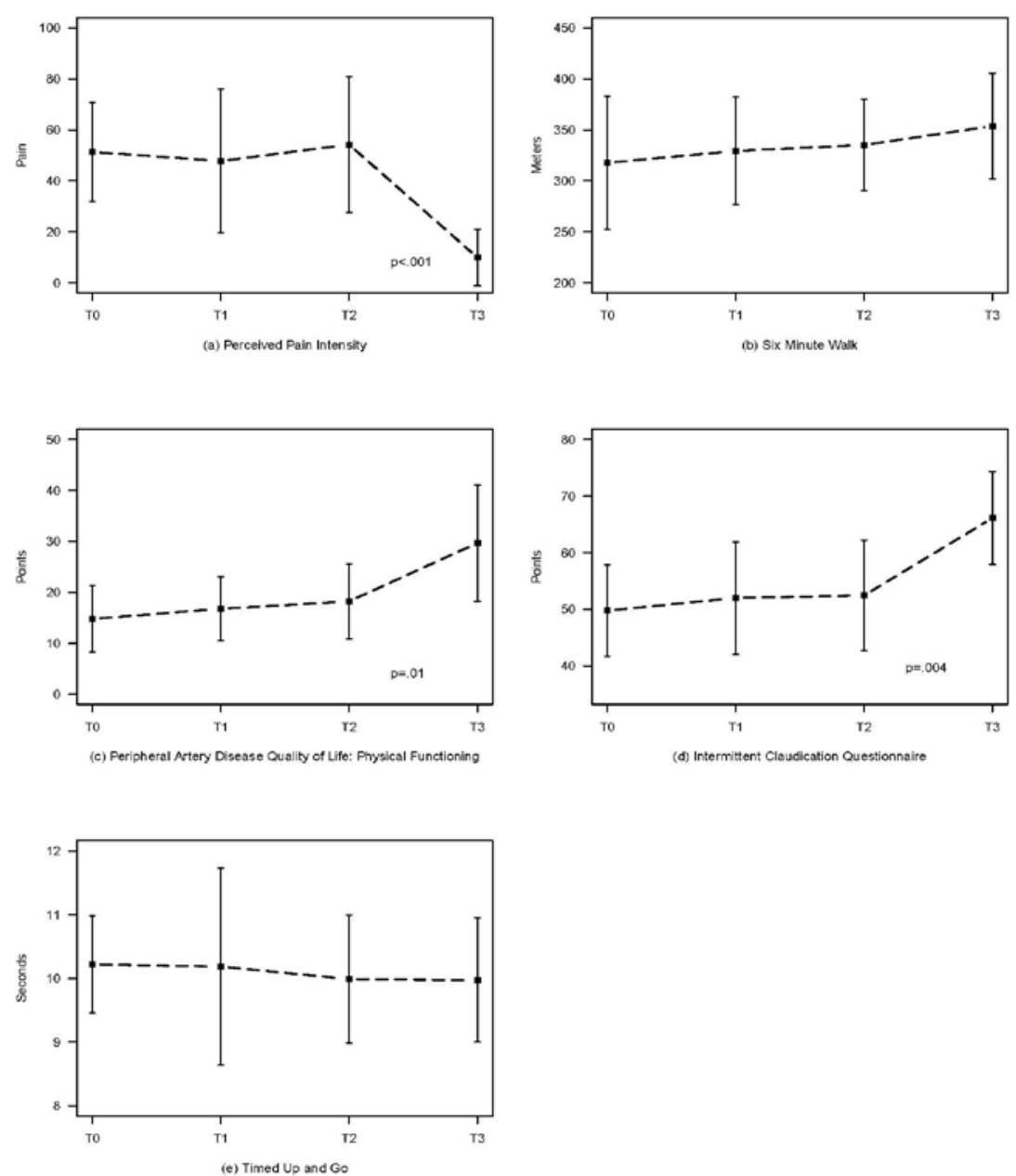

Figure 2: Changes in studied outcome measures over time.

The findings of the repeated measures ANOVA assessing the change over time of the five metrics are likewise summarized in Table 1. As seen, the corrected P-values of PPI, PADQOL, and ICQ ranged from $p=0.01$ to $p=0.0008$. Paired t-tests compared the values measured at $\mathrm{T} 2$ and $\mathrm{T} 3$ confirming that three of the five outcome measures, PPI, PADQOL, and ICQ, all improved significantly as a result of applying FES. The calculated effect size of the outcomes that improved statistically yielded a very large effect on PPI $\left(\eta^{2}=.42\right)$, PADQOL $\left(\eta^{2}=.36\right)$ and ICQ $\left(\eta^{2}=.36\right)$. Figure 2 represents a plot of the 5 outcome measures.

\section{Discussion}

The crossover data added considerable validity to the primary study [17] by confirming that applying FES while walking, can predict a markedly diminished intermittent claudication pain and concurrent improvement of quality of life measures. Combining the patients from the original study $(n=13)$ and the nine patients who crossed over, 20 of 22 patients $(90 \%)$ who walked with FES reported considerable improvement. Ten patients reported that their claudication pain was functionally negligible (0-7 on the scale of $0-100), 6$ patients became mild (less than 20 on the scale) and 4 between 22 and 28 points out of 100. In fact, compared to T2 data, the perceived pain at T3 was $81.7 \%$ lower as a result of walking with FES (Table 1). Direct comparison of the crossover data to published studies' effects on IC pain reduction is not possible because of experimental differences in measuring pain intensity $[18,21]$. However indirect, approximate comparison of success in pain reduction after endovascular intervention reported by Land et al. [22] appears at least comparable to the pain reduction after walking with FES for 8 weeks.

The current data supported the hypothesis that using FES while walking is likely improves quality of life measures, specifically impaired measures due to the severe ischemic pain patients with PAD endure. Improvement of 11.5 points and 13.7 points on the PADQOL-symptom and limitation in physical functioning (factor 3) and ICQ scales respectively, appear 2.2 and 4.7 times greater than when these patients were part of the WALK only group of the original study [17]. These data should remove doubts about the fidelity of FES contribution to improving quality of life measures for patients with PAD and IC $[20,23]$. In the original study we reported that the 4 other factors of the PADQOL questionnaire (Social Relationships, Self-Concepts and Feelings, Fear and Uncertainty, and Positive Adaptation) did not change significantly over time in either the FES+Walk or Walk groups. It is conceivable that these factors of the PAD quality of life questionnaire might be appropriate health-related 
quality of life measures, but do not reflect or interrelate to walking impairment or functional status of PAD patients who are able to ambulate in the home environment [20].

In both the original and crossover periods we elected not to collect mechanistic data such as change in muscle perfusion or changes in collateral circulation due to budgetary constraints. Accordingly, the mechanisms that govern the marked reduction of IC remain unsubstantiated. Studies that applied electrical stimulation to the plantarflexors of patient with $\mathrm{PAD}$ in a stationary position (not FES) [24-26] demonstrated significant improvement in microvessel density and transcutaneous oxygen tension [24], claudicating distance, maximum walking distance, and muscle performance determined by a fatigue index [26]. While not a true proxy, these findings may indirectly help to formulate a hypothesis that 8 weeks of walking with FES may promote an vascular response manifested by improvement of microcirculation leading to better oxygen perfusion in the stimulated muscles. This hypothesis must wait for controlled experimental testing.

In this crossover design, we believe the confounds of carry-over and learning effect were eliminated by data collection of the outcome measure over 16 weeks of walking before adding the FES. Moreover, having a large effect size $\eta^{2}$ ranging between .42 to .36 adds confidence to the treatment effect of FES. Nonetheless, there are few shortcomings of clinical significance. First, the perceived claudication pain remained above $10 \%$ in 11 of the 22 patients at the study endpoint meaning that $50 \%$ were not pain-free, suggesting that 8-weeks intervention was too short. Moreover, it suggests that these patients' peripheral circulation remain inadequate after 8 weeks of walking with FES. Conceivably, increasing the intervention to 3 or more months as done with supervised exercise training [27] may resolve completely the IC and restore adequate peripheral circulation. Second, among the consented patients none presented with critical limb ischemia (CLI) [28] and thus the current findings are not applicable to this subgroup of PAD Third, not having data regarding the number and location of lesions that each participant had in the lower limbs [29] make it difficult to explain why some patient responded better than others to the FES intervention. These shortcomings can be minimized by a modified clinical study that includes detailed arteriograms of the peripheral vascular system, sub-grouping the patients based on occlusion severity, and longer period of FES use. In summary, this crossover study added considerable validity and support to the hypothesis that applying FES while walking can markedly diminish the intermittent claudication pain and improve quality of life measures in community ambulatory patients with PAD.

\section{Acknowledgements}

Research reported in this publication was supported by the National Institute on Aging of the National Institutes of Health under grant number R21AG048001-01.

The content is solely the responsibility of the authors and does not necessarily represent the official views of the National Institutes of Health.

\section{References}

1. Street $\mathrm{T}$, Taylor $\mathrm{P}$, Swain I. Effectiveness of functional electrical stimulation on walking speed, functional walking category, and clinically meaningful changes for people with multiple sclerosis. Archives of physical medicine and rehabilitation. 2015; 96: 667-672.

2. Kafri M, Laufer $Y$. Therapeutic effects of functional electrical stimulation on gait in individuals post-stroke. Ann Biomed Eng. 2015; 43: 451-466.

3. O'Dell MW, Dunning K, Kluding $P$, et al. Response and prediction of improvement in gait speed from functional electrical stimulation in persons with poststroke drop foot. PM R. 2014; 6: 587-601.

4. Kern H, Barberi L, Lofler S, et al. Electrical stimulation counteracts muscle decline in seniors. Frontiers in aging neuroscience. 2014; 6: 189.

5. Springer S, Laufer Y, Becher M, Vatine JJ. Dual-channel functional electrical stimulation improvements in speed-based gait classifications. Clin Interv Aging. 2013; 8: 271-277.

6. Kluding PM, Dunning K, O'Dell MW, et al. Foot drop stimulation versus ankle foot orthosis after stroke: 30-week outcomes. Stroke; a journal of cerebral circulation. 2013; 44: 1660-1669.

7. Danino B, Khamis S, Hemo Y, et al. The efficacy of neuroprosthesis in young hemiplegic patients, measured by three different gait indices: early results. Journal of children's orthopaedics. 2013; 7: 537-542.

8. Embrey DG, Holtz SL, Alon G, Brandsma BA, McCoy SW. Functional electrical stimulation to dorsiflexors and plantar flexors during gait to improve walking in adults with chronic hemiplegia. Archives of physical medicine and rehabilitation. 2010; 91: 687-696.

9. Mann GE, Finn SM, Taylor PN. A pilot study to investigate the feasibility of electrical stimulation to assist gait in Parkinson's disease. Neuromodulation. 2008; 11: 143-149.

10. Alon G, Levitt AF, McCarthy PA. Functional electrical stimulation (FES) may modify the poor prognosis of stroke survivors with severe motor loss of the upper extremity: a preliminary study. Am J Phys Med Rehabil. 2008; 87: 627 636.

11. Taylor PN, Burridge JH, Dunkerley AL, et al. Patients' perceptions of the Odstock Dropped Foot Stimulator (ODFS). Clin Rehabil. 1999; 13: 439-446.

12. Bethoux F, Rogers HL, Nolan KJ, et al. Long-Term Follow-up to a Randomized Controlled Trial Comparing Peroneal Nerve Functional Electrical Stimulation to an Ankle Foot Orthosis for Patients With Chronic Stroke. Neurorehabil Neural Repair. 2015; 29: 911-922.

13. Strasser EM, Stattner S, Karner J, et al. Neuromuscular electrical stimulation reduces skeletal muscle protein degradation and stimulates insulin-like growth factors in an age- and current-dependent manner: a randomized, controlled clinical trial in major abdominal surgical patients. Ann Surg. 2009; 249: 738-743.

14. Benavent-Caballer V, Rosado-Calatayud $P$, Segura-Orti E, Amer-Cuenca $\mathrm{JJ}$, Lison JF. Effects of three different low-intensity exercise interventions on physical performance, muscle CSA and activities of daily living: a randomized controlled trial. Exp Gerontol. 2014; 58: 159-165.

15. Wall BT, Dirks ML, Verdijk LB, et al. Neuromuscular electrical stimulation increases muscle protein synthesis in elderly type 2 diabetic men. American journal of physiology. Endocrinology and metabolism. 2012; 303: E614-623.

16. Miyamoto T, Fukuda K, Kimura T, Matsubara Y, Tsuda K, Moritani T. Effect of percutaneous electrical muscle stimulation on postprandial hyperglycemia in type 2 diabetes. Diabetes Res Clin Pract. 2012; 96: 306-312.

17. Embrey DG, Alon G, Brandsma BA, et al. Functional electrical stimulation improves quality of life by reducing intermittent claudication. Int $\mathrm{J}$ Cardiol. 2017; 243: 454-459.

18. Addison O, Ryan AS, Prior SJ, et al. Changes in Function After a 6-Month Walking Intervention in Patients With Intermittent Claudication Who Are Obese or Nonobese. J Geriatr Phys Ther. 2016; 40: 192-196.

19. Treat-Jacobson D, Lindquist RA, Witt DR, et al. The PADQOL: development and validation of a PAD-specific quality of life questionnaire. Vasc Med. 2012; 17: 405-415.

20. Conijn AP, Jens S, Terwee CB, Breek JC, Koelemay MJ. Assessing the quality of available patient reported outcome measures for intermittent 
claudication: a systematic review using the COSMIN checklist. Eur J Vasc Endovasc Surg. 2015; 49: 316-334.

21. Dipnarine K, Barak S, Martinez CA, Carmeli E, Stopka CB. Pain-free treadmill exercise for patients with intermittent claudication: Are there gender differences? Vascular. 2016; 24: 304-314.

22. Lang PM, Vock G, Schober GM, et al. Impact of endovascular intervention on pain and sensory thresholds in nondiabetic patients with intermittent claudication: a pilot study. J Pain. 2009; 10: 264-273

23. Guidon $M, M c G e e H$. One-year effect of a supervised exercise programme on functional capacity and quality of life in peripheral arterial disease. Disabil Rehabil. 2013; 35: 397-404.

24. Clover AJ, McCarthy MJ, Hodgkinson K, Bell PR, Brindle NP. Noninvasive augmentation of microvessel number in patients with peripheral vascular disease. J Vasc Surg. 2003; 38: 1309-1312.

25. Debreceni L, Gyulai M, Debreceni A, Szabo K. Results of transcutaneous electrical stimulation (TES) in cure of lower extremity arterial disease. Angiology. 1995; 46: 613-618.
26. Tsang GM, Green MA, Crow AJ, et al. Chronic muscle stimulation improves ischaemic muscle performance in patients with peripheral vascular disease. European journal of vascular surgery. 1994; 8: 419-422.

27. Gardner AW. Exercise rehabilitation for peripheral artery disease: An exercise physiology perspective with special emphasis on the emerging trend of home-based exercise. Vasa. 2015; 44: 405-417.

28. Reinecke $\mathrm{H}$, Unrath $\mathrm{M}$, Freisinger $\mathrm{E}$, et al. Peripheral arterial disease and critical limb ischaemia: still poor outcomes and lack of guideline adherence. Eur Heart J. 2015; 36: 932-938.

29. Smolderen KG, van Zitteren M, Jones PG, et al. Long-Term Prognostic Risk in Lower Extremity Peripheral Arterial Disease as a Function of the Number of Peripheral Arterial Lesions. J Am Heart Assoc. 2015; 4: e001823.
Phys Med Rehabil Int - Volume 4 Issue 5 - 2017

ISSN : 2471-0377 | www.austinpublishinggroup.com

Alon et al. (@ All rights are reserved
Citation: Alon G, Embrey DG, Brandsma BA, Vladimir F, Silva A, Pflugeisen BM, et al. Functional Electrical Stimulation (FES) Reduces Intermittent Claudication (IC) and Improves Quality of Life (QOL) Measures: A Cohort Crossover. Phys Med Rehabil Int. 2017; 4(5): 1130. 